\title{
Correction to: Evaluation of the bioequivalence and food effect on the bioavailability of CC-486 (oral azacitidine) tablets in adult patients with cancer
}

\author{
Hani M. Babiker ${ }^{1}$ (1) - Mohammed Milhem ${ }^{2}$. Joseph Aisner ${ }^{3} \cdot$ William Edenfield $^{4}$. Dale Shepard ${ }^{5} \cdot$ Michael Savona $^{6}$. \\ Swaminathan Iyer ${ }^{7} \cdot$ Maen Abdelrahim $^{8} \cdot$ C. L. Beach ${ }^{9} \cdot$ Barry Skikne $^{9,10} \cdot$ Eric Laille $^{9} \cdot$ Kao-Tai Tsai $^{9} \cdot$ Thai Ho $^{11}$
}

Published online: 25 March 2020

(c) Springer-Verlag GmbH Germany, part of Springer Nature 2020

\section{Correction to: Cancer Chemotherapy and Pharmacology (2020) 85:621-626 \\ https://doi.org/10.1007/s00280-020-04037-9}

In the original publication of the article the author has found few incorrect values in the Table 1 . The corrected Table 1 is given below.

\footnotetext{
The original article can be found online at https://doi.org/10.1007/ s00280-020-04037-9.

Hani M. Babiker

hanibabiker@email.arizona.edu

University of Arizona Comprehensive Cancer Center,

Tucson, AZ, USA

2 University of Iowa Hospital, Iowa City, IA, USA

3 Rutgers Cancer Institute of New Jersey, New Brunswick, NJ, USA

4 Greenville Health System, Greenville, SC, USA

5 Cleveland Clinic, Cleveland, OH, USA

6 Vanderbilt University Medical Center, Nashville, TN, USA

7 MD Anderson Cancer Center, Houston, TX, USA

8 Houston Methodist Hospital, Houston, TX, USA

9 Celgene Corporation, Summit, NJ, USA

10 University of Kansas Medical Center, Kansas City, KS, USA

11 Mayo Clinic, Phoenix, AZ, USA
} 
Table 1 Food effect on the plasma pharmacokinetic parameters of the CC-486 $300 \mathrm{mg}$ Formulation B tablet

\begin{tabular}{|c|c|c|c|c|c|c|c|c|c|c|}
\hline \multirow{2}{*}{$\begin{array}{l}\text { Condition } \\
\text { Fasted }\end{array}$} & \multicolumn{2}{|c|}{ Statistic } & \multicolumn{2}{|c|}{ AUCt (ng h/mL) } & $\mathrm{AUC}_{\infty}(\mathrm{ng} \mathrm{h} / \mathrm{mL})$ & $C_{\max }(\mathrm{ng} / \mathrm{mL})$ & $T_{\max }(\mathrm{h})$ & $t_{1 / 2}(\mathrm{~h})$ & $\mathrm{CL} / \mathrm{F}(\mathrm{L} / \mathrm{h})$ & $\mathrm{Vz} / \mathrm{F}(\mathrm{L})$ \\
\hline & \multicolumn{2}{|l|}{$N$} & \multicolumn{2}{|l|}{56} & 54 & 56 & 56 & 54 & 54 & 54 \\
\hline & \multicolumn{2}{|c|}{ Geometric Mean $^{a}$} & \multicolumn{2}{|l|}{224.9} & 234.5 & 131.7 & NA & 0.58 & 1280 & 1074 \\
\hline & \multicolumn{2}{|c|}{ Geometric \% CV } & \multicolumn{2}{|l|}{68.4} & 62.9 & 70.4 & NA & 31.6 & 62.9 & 76.2 \\
\hline & \multicolumn{2}{|c|}{ Median } & \multicolumn{2}{|l|}{240} & 243 & 141 & 1.0 & 0.55 & 1230 & 1140 \\
\hline & \multicolumn{2}{|c|}{ Min, $\max$} & \multicolumn{2}{|c|}{$41.5,1050$} & $65.9,1050$ & $21.4,608$ & $0.50,4.0$ & $0.35,1.7$ & 286,4550 & 228,6310 \\
\hline \multirow[t]{5}{*}{ Fed } & \multicolumn{2}{|l|}{$N$} & \multicolumn{2}{|l|}{55} & 44 & 55 & 55 & 46 & 44 & 44 \\
\hline & Geol & etric Mean ${ }^{\mathrm{a}}$ & 233.7 & & 248.9 & 105.2 & NA & 0.78 & 1205 & 1279 \\
\hline & Geor & etric \%CV & 58.3 & & 52.0 & 76.3 & NA & 55.2 & 52.0 & 80.5 \\
\hline & Med & & 250 & & 250 & 116 & 2.0 & 0.68 & 1200 & 1160 \\
\hline & Min, & Max & 61.3 & & $78.9,560$ & $22.7,337$ & $0.50,6.1$ & $0.40,6.2$ & 536,3800 & 399,9350 \\
\hline PK parame & & Condi & & $N$ & Geometric mean $^{\dagger}$ & $\begin{array}{l}\text { Ratio (\% } \\
\text { ric mean }\end{array}$ & of geomet- & $\begin{array}{l}90 \% \mathrm{CI} \text { o } \\
\text { geometric }\end{array}$ & $\begin{array}{l}\text { atio }(\%) \text { of } \\
\text { heans }\end{array}$ & $\begin{array}{l}\text { Intra- } \\
\text { patient } \\
\% \mathrm{CV}\end{array}$ \\
\hline $\mathrm{AUC}_{\mathrm{t}}(\mathrm{ng} \mathrm{h}$ & $\mathrm{mL})$ & Fasted & & 56 & 227.8 & 101.7 & & $(92.83,11$ & & 29.0 \\
\hline & & Fed & & 55 & 231.8 & & & & & \\
\hline $\mathrm{AUC}_{\infty}$ (ng & $/ \mathrm{mL})$ & Fasted & & 54 & 237.5 & 108.9 & & $(98.48,12$ & & 28.3 \\
\hline & & Fed & & 44 & 258.7 & & & & & \\
\hline$C_{\max }(\mathrm{ng} / \mathrm{m}$ & & Fasted & & 56 & 133.0 & 78.86 & & $(68.58,9$ & & 45.5 \\
\hline & & Fed & & 55 & 104.9 & & & & & \\
\hline PK parame & & Condition & $N$ & Median & Median diffe & ence & CI of media & difference & Fed-fasted) & $P$ value \\
\hline $\operatorname{Tmax}(h)^{\ddagger}$ & & Fasted & 56 & 1.0 & 1.0 & $(0.7$ & 1.3) & & & $<0.001$ \\
\hline & & Fed & 55 & 2.0 & & & & & & \\
\hline
\end{tabular}

$N$ patients $=$ the total number of patients for which the PK parameter could be calculated. $\mathrm{AUC}_{\infty}, \mathrm{t}_{1 / 2}, \mathrm{CL} / \mathrm{F}$, and V/F could not be calculated in some instances, if there were not data from a sufficient number of time points after the occurrence of $C_{\max }$

Slight differences in geometric means of $\mathrm{AUC}_{\infty}$ and $C_{\max }$ are due to different methods of calculation

$A U C_{t}$ area under the plasma concentration-time curve from time 0 to time $t, A U C_{\infty}$ area under the plasma concentration-time curve from time 0 extrapolated to infinity, $C_{\max }$ maximum plasma concentration, $T_{\max }$ time to $\mathrm{C}_{\max }, t_{1 / 2}$ terminal half-life, $C L / F$ apparent total clearance, $V z / F$ volume of distribution, $N A$ not applicable

${ }^{\dagger}$ Calculated using an analysis of variance (ANOVA) model

"Median and median difference (Test vs. Reference), and 90\% CI of the median difference, are from Hodges-Lehmann estimate. The $P$ value is from Wilcoxon signed-rank test

${ }^{a}$ Calculated using summary statistics.

Publisher's Note Springer Nature remains neutral with regard to jurisdictional claims in published maps and institutional affiliations. 